\title{
Vertical scanning biases and their possible influence on reading direction: Celtic wisdom or folly?
}

\author{
ALEJANDRA M. AMENGUAL, ${ }^{1,2}$ VALERIA DRAGO,,${ }^{1,3}$ PAUL S. FOSTER, ${ }^{1}$ \\ RAMON C. LEIGUARDA, ${ }^{2}$ AND KENNETH M. HEILMAN ${ }^{1}$ \\ ${ }^{1}$ Department of Neurology, University of Florida and Malcom Randall Veterans Affairs Medical Center, Gainesville, Florida \\ ${ }^{2}$ Raúl Carrea Institute of Neurological Research, FLENI, Buenos Aires, Argentina \\ ${ }^{3}$ Oasi Institute for Research on Mental Retardation and Brain Aging, Troina, Enna, Italy \\ (Received March 27, 2007; Final Revision July 23, 2007; AcCePted July 23, 2007)
}

\begin{abstract}
The reason people read from top to bottom is unknown, but could be related to brain-mediated directional biases or environmental factors. To learn if there is a brain-mediated directional bias responsible for top-down reading direction, we evaluated the directional scanning in the vertical dimension by using directional letter and face cancellation tasks. Twenty participants were instructed to cancel either target letters or faces using either an up-down or down-up direction, with the stimuli located in left, right, and center hemispace. The results indicated significant differences in completion time between the search direction (up vs. down) and spatial position for the letter cancellation task, with a faster completion time for the bottom-up scan in right space and top-down in left space. Because the left hemisphere primarily attends to contralateral right hemispace our results suggest that, when attending to letter stimuli, the left hemisphere is biased to scan in a proximal to distal (upward) direction. Although the reasons why this is reversed in left hemispace and why we did not see directional biases in the face condition remains unclear, these results do suggest that the direction in which we learn to read is inconsistent with the brain's intrinsic directional bias. (JINS, 2008, 14, 102-109.)
\end{abstract}

Keywords: Visuospatial attention, Letters cancellation task, Faces cancellation task, Scanning direction, Attentional bias, Focal attention

\section{INTRODUCTION}

While in some languages people read from right to left and in others from left to right, in all current written languages people read from the top to the bottom. The reason for this top down bias is not known, but might be related to the organization of the human brain. Most traditional information processing models of brain functions posit a series of anatomically focal modules that contain representations and process information. These modules receive and send information to other modules allowing complex tasks to be accomplished. For example, the process of "reading" requires the interaction between several different networks. The first step is orienting visuospatial attention toward the page con-

Drs. Amengual and Drago contributed equally to this work.

Correspondence and reprint requests to: Valeria Drago, University of Florida, Department of Neurology and Center for Neuropsychological Studies, McKnight Brain Institute at UF, 100 Newell Drive, Room L3-1001, Gainesville, FL 32610-0236. E-mail: valeria.drago@neurology.ufl.edu taining the print. Subsequently, visual systems process the shapes of the letters until the percepts of the letter are formed. After the letters are perceived, there are several systems that can be used to read (Coslett, 2003). When learning to read, most children use a system where letters or groups of letter (graphemes) are translated to their speech sounds (phonemes). This pattern of sounds, like heard speech can then access the phonological lexicon and semantic networks, thereby allowing a person to derive meaning or to pronounce-say the written word (see Figure 1).

With regard to the first step in reading, the spatial orienting of attention to the top of the page, studies have revealed that normal people do have spatial biases. However, it is unclear if this initial orientation to the top of the page is based on an intrinsic brain-based attentional scanning bias or is learned. A well-established paradigm that allows investigators to study how subjects orient their attention in space is the line bisection task. In this simple task, subjects are required to bisect a line centered on a sheet of paper. If 


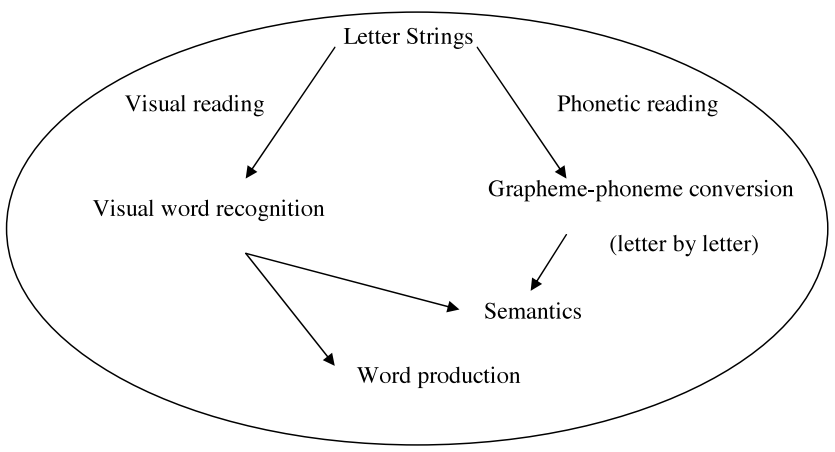

Fig. 1. Diagram of a model of the reading system depicting possible routes for reading: grapheme converted to phoneme reading (letter by letter), direct visual recognition of the entire word. The latter route may or may not have access to semantic representations.

subjects demonstrate systematic mis-bisections, a directional bias, this bias indicates that there is an asymmetrical spatial allocation of attention in the direction of the attempted bisection. People usually read in one of two planes, the transverse plane, below eye level and the coronal plane, which is perpendicular to the transverse plane. To determine whether normal people have attentional biases in these two planes, several studies have been performed using the line bisection task where the lines were placed in these planes (intersection of the midsagittal and coronal as well as the intersection of the midsagittal and transverse planes). The results of these studies demonstrate that vertical lines are often mis-bisected above the true center (Shelton et al., 1990), and radial lines, below eye level, were mis-bisected distal to the true center (Geldmacher \& Heilman, 1994; Halligan \& Marshall, 1993; Shelton et al., 1990). This up or distal bias might help explain why people start reading from the top of the paper.

To explain these biases Jeerakathil and Kirk (1994) introduced the concept of an object-centered bias in which visual attention is always biased toward the top of an object, for example the top of a page. In contrast, Geldmacher and Heilman (1994) suggested that people have a viewercentered bias that could be either retinotopic (attentional bias for stimuli detected in the upper visual field) or body centered (attentional bias for far peripersonal space). To help learn if this radial bias is object centered, retinotopic or body centered, normal subjects attempted to bisect radial lines both above and below eye level. When radial lines are below eye level the top of the page is distal, but when above eye level the top of the page is proximal. The results of this study indicated that, when radial lines were placed below eye level, there was a distal bias, but this bias was no longer present above eye level. If the bias was entirely object centered, we would expect a bias toward the top of the object either above or below the eyes level. If the bias, in contrast, were retinotopic, it would have reversed from distal to proximal. The results that radial lines below eye level are bisected distally and when they are above eye level this bias decreases suggest that, when the page is below eye level, the distal attentional bias might be induced by a combination of bodycentered and retinotopic attentional biases, whereas when the line is above eye level, the proximal portion of the line falls in the lower part of the retina (upper visual field), resulting in a "relative proximal" bias (see Figure 2).

Based on this research, it is possible that people start reading from the top-distal portion of the page because they have an intrinsic retinotopic and body-centered attentional bias. There is, however, another visual attentional bias that might also influence peoples' allocation of attention when reading. Using a perceptual bisection task, similar to the Landmark test (Harvey et al., 1995), Heilman et al. (1995) demonstrated that, when the radially oriented lines were in the right hemispace, and thus primarily attended to by the left hemisphere, there is a proximal attentional bias. In contrast, when these radial lines are placed in the left hemispace and, thus, attended to primarily by the right hemisphere, there was a distal bias. Positron emission tomography has subsequently provided converging evidence that bisections in near space lead to left hemispheric activation and bisections in far space induce right hemisphere activation (Weiss et al., 2000). Because reading is primarily performed by the left hemisphere, activation of the left hemisphere might bias attention to the proximal portion of the page. Support for the proximal bias postulate was provided by Jeong et al. (2006), who had normal subjects attempt to bisect radially oriented lines that were solid or composed of symbolic characters. In the latter symbolic task, the subjects had to cancel through a given symbol that was closest to the midline. In addition to processing letter and symbols, the left hemisphere appears to be dominant for mediating focal attention (Robertson et al., 1988). Thus, increasing the demands for focused attention and using symbols both induce greater left than right hemisphere activation, and when compared with the solid line bisection, this character line bisection was associated with a proximal bias. These studies suggest that, when a person is reading, his or her attention would normally be biased to the more proximal portion of the page and that the practice of reading from distal (top) to proximal (bottom) might have been influenced by other factors.

Although the line bisection task is excellent for assessing attentional biases and has been extensively used in the clinic and in research, the cancellation test is also frequently used to assess for certain aspects of spatial neglect, including hemispatial unawareness, motor perseveration, and deficits of manual and visual exploration. Whereas the line bisection is excellent for assessing spatial attentional biases, even in normal subjects, the cancellation test is, in general, not ideal for this purpose with almost all normal subjects being able to cancel all the target stimuli. In this study, however, our primary interest was to assess the efficiency of different (top-down $v s$. bottom-up) search strategies and while spatial attentional biases can influence spatial search strategies, the cancellation test, which can be timed, is a better tool to assess the efficiency of different search strategies and, thus, was used for this study. 


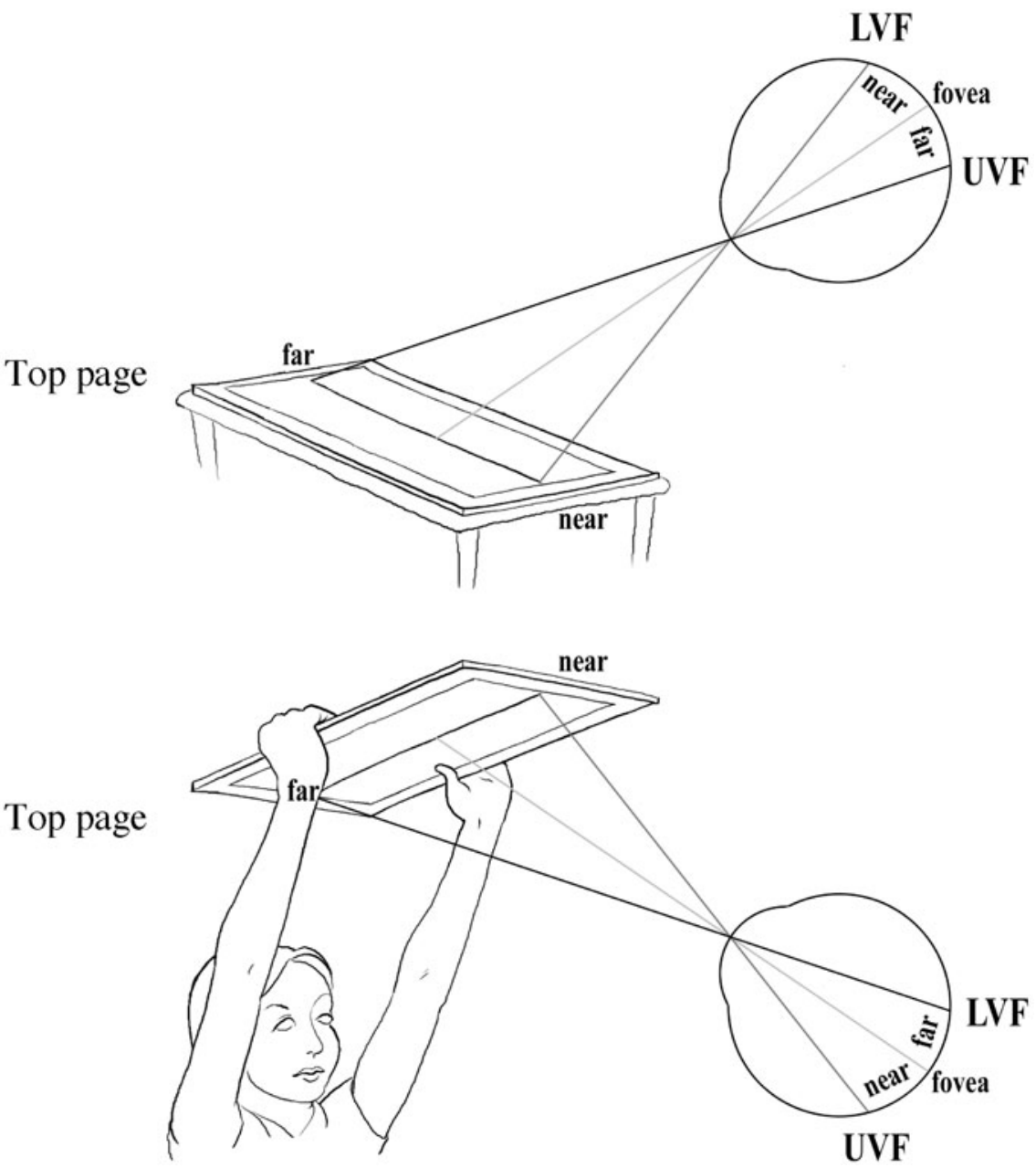

Fig. 2. This figure shows how, when radial lines are below eye level, the top of the page is distal, but when above eye level, the top of the page is proximal.
The main goal of this study was to learn if, when scanning for letters and faces, normal people have a propensity to initially allocate attention distally and then move their attention proximally (top to bottom) versus initially allocating attention proximally and moving it distally (bottom to top). To assess the relative efficiency of directional allocation of attention in the radial, below eye level, dimension we used directional (up-down; down-up) letter and face cancellation tasks. To better understand the possible difference between right and left hemispheric influence on the allocation of attention, we performed these cancellation tasks in three portions of the viewer-centered space (center, right, and left). We assumed that there would be interactions between-stimulus type (faces $v s$. letters) and hemispace (left and right) such that the normal subjects would be more efficient when processing faces in left compared with right hemispace and would be more efficient processing letters in right than left hemispace. Because the left hemisphere has a proximal bias and the right hemisphere a distal bias, we also expected that, in right hemispace, a bottom-up search would be more efficient (faster) than top-down (especially in the letter condition), and in left hemispace a top-down search (especially with faces) would be more efficient (faster) than a bottom-up search.

\section{METHODS}

\section{Participants}

A total of 20 healthy volunteers (10 men and 10 women; mean age, $30.5 \pm 7.6$ years) with no history of neurological or psychiatric illness were recruited. All subjects were righthanded as assessed by the Edinburgh handedness inventory. Before the experiment, they underwent a complete neurological evaluation and all subjects were normal. Any human data included in this manuscript was obtained in compliance with the Helsinski Declaration.

\section{Apparatus}

We developed two cancellation tasks.

\section{Faces cancellation task}

Stimuli consisted of 12 target faces and 72 foil faces ( 84 total faces). These nonfamiliar faces were $12 \times 16 \mathrm{~mm}$ black and white pictures, all men with similar features (age, race) and all had a neutral expression (Figure 3). For each trial, one of the faces was selected as the target face. These 


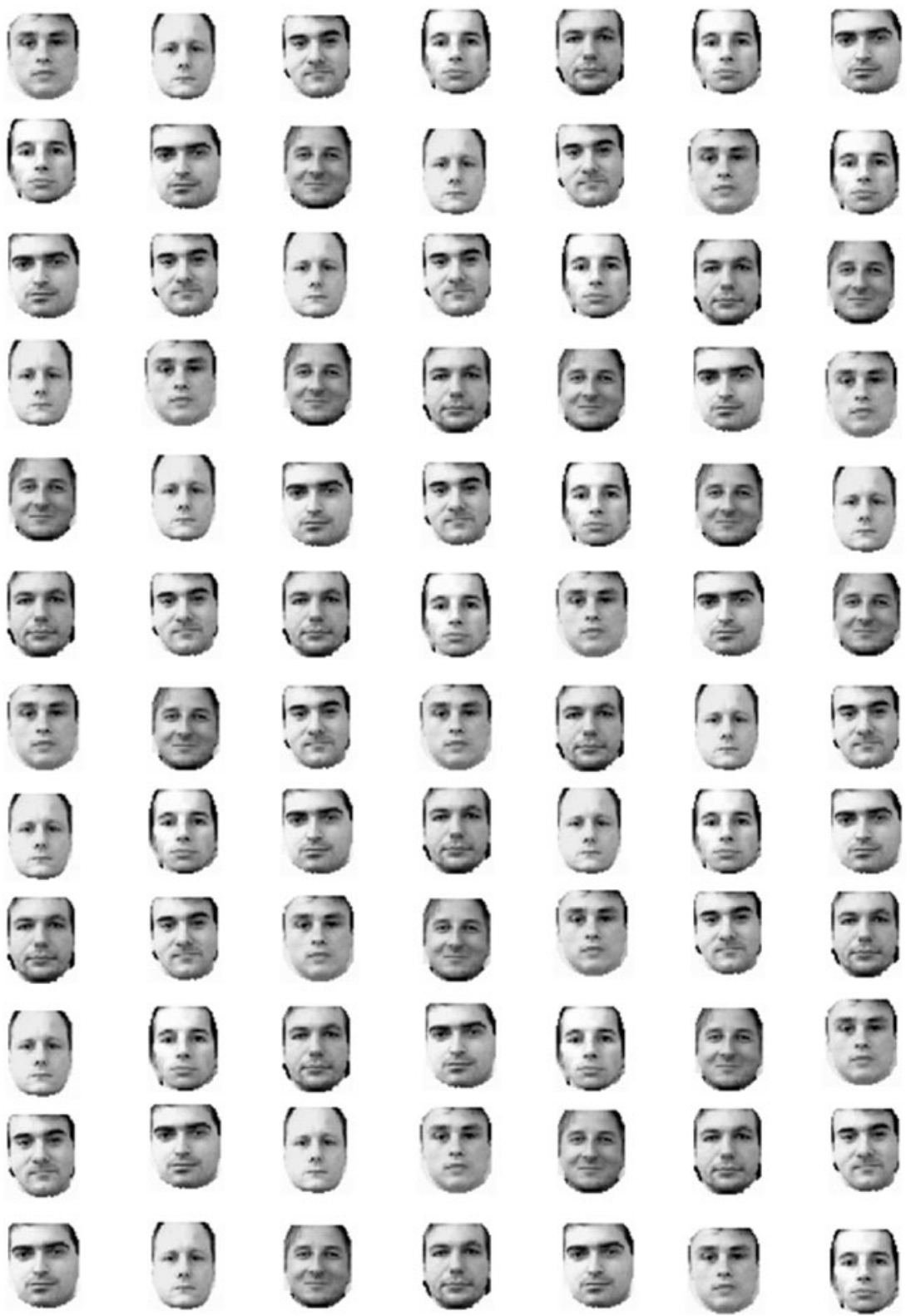

Fig. 3. Faces cancellation task.

faces were placed in a structural array of 7 columns and 12 lines on a $(210 \times 297 \mathrm{~mm})$ sheet of paper. Targets were randomly placed on the sheet, but there were an equal number of targets in each quadrant.

\section{Letters cancellation task}

Letters (b, p, d, and g) were presented on sheets of paper that were $420 \times 297 \mathrm{~mm}$, and these letters were placed in a structured array of 12 columns and 16 lines. One of the letters was selected as the target letter, and on each sheet, there were 24 target letters and 168 distracting (nontarget) stimuli. The targets were randomly distributed on the sheet, but the 24 targets were equally distributed in each quadrant. We used different fonts with either capitals or lower case to help ensure that letters were recognized as graphemic sym- bols (representing a phoneme) and not just identified by their visual similarity (Figure 4).

\section{Procedures}

The subjects were seated in a chair, directly in front of a table, and were allowed to move their eyes or head but not their torso. They were given a pencil and instructed to use their right hand to cancel all the targets they could find. For the faces cancellation task, they were given a demonstration sheet with one sample of each face where the target was circled. In our study, the face and letter cancellation tasks were not entirely parallel. These two tasks had a different number of target stimuli; thus, the sizes of the sheets of papers were different, and there were more foils in the letter than in the face cancellation task. Additionally, in the 


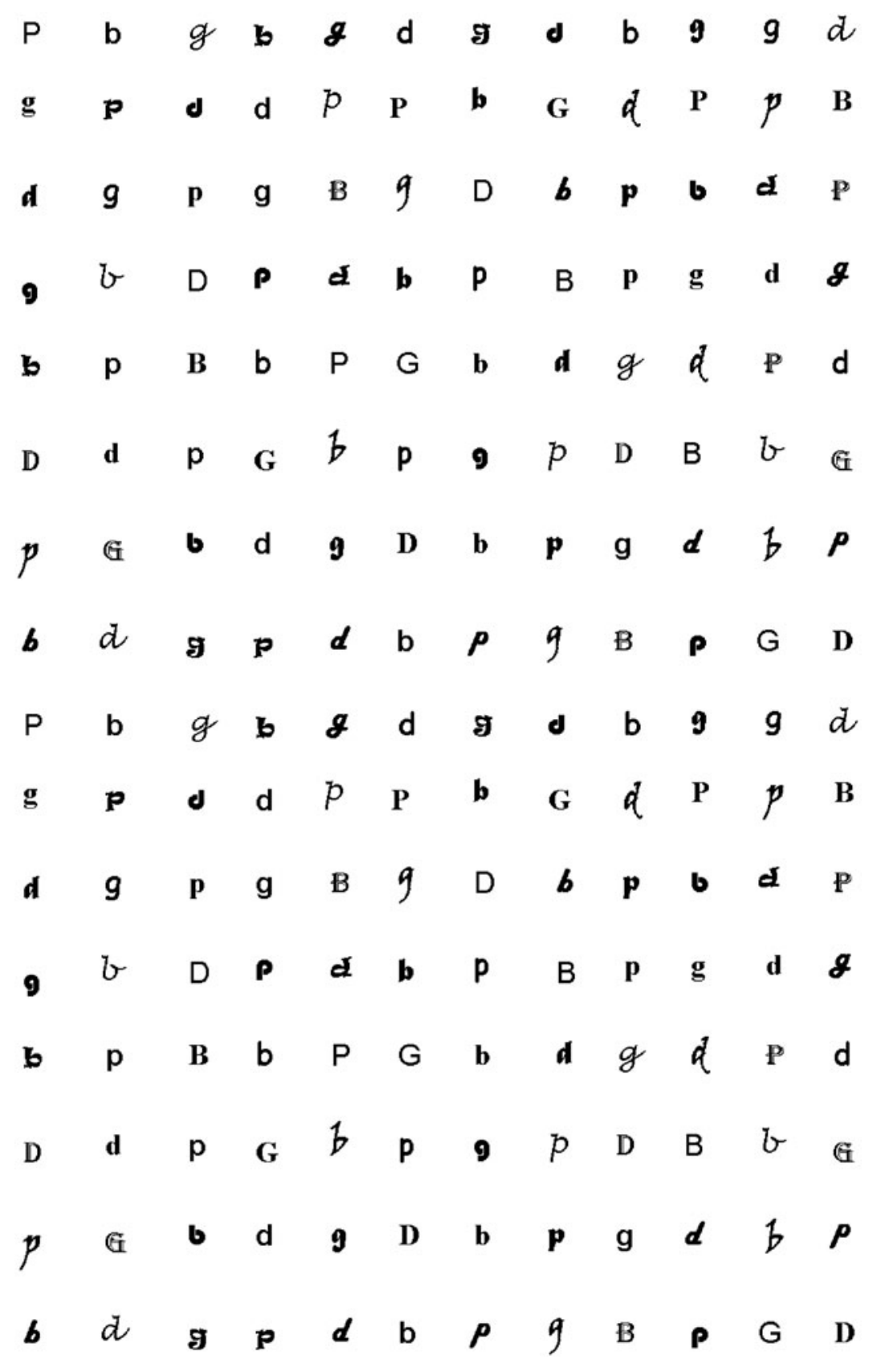

Fig. 4. Letters cancellation task. letter cancellation task, subjects had to detect a sequence of two letters. In pilot observations, the subjects thought that detecting isolated letters was easier that detecting target faces, and we altered the letter detection task in attempt to make detection equally difficult.

Our result demonstrated that, for our experimental subjects, the time required to complete the faces tasks was not significantly different than the time it took to complete the letters tasks. Additionally, our primary interest was learning if each cancellation task (face and letter) was better performed in a top-down or a bottom-up scan, and learning if the hemispace placements would influence the subjects' performances in these two different cancellation tasks (face and letter).
For the letters cancellation task, the subjects were verbally instructed to find a target letter, but only if this letter was preceded in the array by another letter specified by the examiner (e.g., "Cancel all Xs only if they are preceded by the letter Y"). Subjects were instructed to perform the search in a vertical direction, following the columns. They were specifically instructed before each test if the search had to be done in a downward direction (from the top of a column down to its end at the bottom and then to proceed the top of the next column) or upward direction (beginning from the bottom of the column). They could start their scanning either from the left or right edge of the sheet of paper.

These cancellation tests were performed in three spatial locations: In the center position, the sheet of paper was 
placed such that the subjects' midsagittal plane went through the center of the paper. In the right and left conditions, the medial edge of the sheet of paper was placed in the sagittal plane that passes along the lateral side of subject's shoulder. Thus, there were 12 different testing conditions, 6 for the faces and 6 for the letters, including downward-center, downward-left, downward-right, upward center, upwardleft, upward-right. The order in which these tests were given was randomized.

\section{RESULTS}

The dependent measure consisted of the time (in seconds) required to complete the tasks (see Table 1). A 2 (Stimulus: Faces and Letters) $\times 2$ (Direction: Upward and Downward) by (Position: Left Hemispace, Center Hemispace, and Right Hemispace) repeated-measures analysis of variance (ANOVA) was used to analyze the data. The results indicated a significant Stimulus $\times$ Direction $\times$ Position interaction $[F(2,38)=5.76 ; p=.007]$. Multiple comparisons were conducted using a Bonferroni correction $(p<.004)$ for experiment wise error rate. These additional analyses indicated a significantly $[t(19)=-4.46 ; p=.0003]$ faster task completion time when using a downward scanning direction with Letter stimuli placed in Center Hemispace $(M=$ 107.09; $S D=19.05$ ) than when using the same scanning direction with Letter stimuli placed in Right Hemispace $(M=121.39 ; S D=24.10)$. Additionally, a significantly $[t(19)=21.98 ; p=.002]$, faster completion time was noted when using an upward scanning direction with Letter stimuli placed in Right Hemispace $(M=107.57$; $S D=16.47)$ than when using a downward scanning direction with Letter stimuli placed in this same hemispace $(M=121.39$; $S D=24.10)$. All other comparisons were not significant. The preferential horizontal starting position in all three hemispatial conditions and with both the face and letter stimuli

Table 1. Reaction time means and standard deviations for the different conditions

\begin{tabular}{lrc}
\hline \hline Condition & Mean & Standard deviation \\
\hline FD Left & 39.0 & 8.9 \\
FD Ce & 38.7 & 6.6 \\
FD R & 41.4 & 9.7 \\
FU Left & 42.3 & 11.4 \\
FU Ce & 38.8 & 9.2 \\
FU R & 41.0 & 9.2 \\
LD Left & 107.7 & 18.6 \\
LD Ce & 107.0 & 19.0 \\
LD R & 121.3 & 24.0 \\
LU Left & 116.8 & 25.4 \\
LU Ce & 109.0 & 17.8 \\
LU R & 107.5 & 16.7 \\
\hline \hline
\end{tabular}

Note. $\mathrm{F}=$ Faces; $\mathrm{L}=$ Letters; $\mathrm{D}=$ Scanning direction Downward; $\mathrm{U}=$ Scanning direction Upward; $\mathrm{Ce}=$ Test Location Center; $\mathrm{R}=$ Test Location Right hemispace; Left $=$ Test Location Left hemispace. was on the left side of the page. The chosen horizontal direction neither affected the results nor interacted with any other manipulations.

We also analyzed the data for the number of omission and commission errors, for both the face and letter stimuli. The results of four separate repeated-measures ANOVAs indicated no significant main effects or interactions for any of these dependent measures.

\section{DISCUSSION}

As mentioned, currently in all languages, people read from the distal portion of a page (top) to the proximal portion of the page (bottom). Although this vertical pattern of reading might be related to brain-based attentional biases, it is also possible that the vertical direction in which people read is influenced by other factors. In our study, all the participants were readers of European languages and, therefore, read from left to right and from top to bottom. Based on this reading behavior, we expected that subjects would be more efficient scanning letters from distal (top) to proximal (bottom) than vice versa. However, our results seem to indicate that healthy individuals, when scanning letters in the right hemispace, are faster (more efficient) when scanning from bottom to top. The first step in word recognition is letter recognition and grapheme to phoneme transcoding. Thus, our finding might suggest that learning to read text from bottom up might be more efficient than the top-down mean that is now the standard.

The explanation for the efficiency of this right hemispace bottom to top grapheme search strategy might be related to several interactive factors, including that the left hemisphere is dominant for grapheme recognition (Cohen et al., 2004) and grapheme to phoneme transcoding, as well as having a propensity to attend to the right and proximal space viewer-centered space (Heilman et al., 1995; Jeong et al., 2006).

Our study was performed by having subjects perform these tasks exclusively with their right hand. Our finding that there were hemispatial differences might be related not to differences in the influence of hemispheric attentional biases, but rather to motor-postural differences induced by using the right hand and arm in right versus left hemispace. Although we do not entirely dismiss the possibility that the spatial placement of the stimuli could have influenced our results in the letter cancellation task, when performing the face cancellation task, subjects used the same postures and movements. Furthermore, because we did not find any hemispace effect on the face cancellation task we suspect that the postural-action differences that occur when a subject works in left versus right hemispace could not account for our results in the letter cancellation task.

When normal subjects bisect lines, they orient their eyes first to one end of the line then scan the entire line from one end to another. After making this scan, normal people direct their eyes to the center of the line before making their bisection. Line bisection is a spatial task that requires the use of 
global ( $v s$. focal) attention and requires continuous ( $v s$. categorical) processing. Studies in normal subjects have demonstrated that it is the right hemisphere that is dominant for global attention (Robertson et al., 1988), continuous processing (Arruda et al., 1996, 2007), and spatial computations (Jager \& Postma, 2003). Thus, when performing the routine line bisection task, normal subjects should initially orient to the left side of the line. When spatial orienting during the performance of the line bisection task was examined, it was found that most normal subjects do have an overall left-sided bias on this task (Bowers \& Heilman, 1980; McCourt et al., 2001). People usually move their eyes in the direction to which they are attending. When performing the line bisection task, the majority of normal subjects initially orient to the left side of the line and then move their attention left to right. In contrast to performing this spatial line bisection task, which induces right hemisphere activation, most people when performing a letter cancellation task activate their left hemisphere. This left hemisphere activation might be induced by the requirement for using left hemisphere-mediated focal ( $v s$. global) attention (Barrett et al., 1998; Lamb \& Robertson, 1990). In addition, as previously mentioned, the letter stimuli also activate the left more than the right hemisphere (Bryden \& Allard, 1976). This left hemisphere activation induces an attentional bias toward right space. Support for this hypothesis comes from several studies (Lee et al., 2004; Mohr \& Leonards, 2007). In these studies, normal subjects performed a letter-line bisection task, which requires reading letters, categorical processing, and focused attention functions all mediated primarily by the left hemisphere. In this letter-line bisection, normal subjects demonstrated a rightward bisection bias. Because left hemisphere activation induces a rightward attentional bias, as well as a viewercentered proximal attentional bias, scanning letters from right to left and proximal (down) to distal (up) might also be more compatible with the brain's attentional biases during letter by letter reading.

Unlike the European languages, people who read Semitic languages read from right to left, and the observation that not all languages are read in the same direction provides additional support for the postulate that the direction in which people read has been influenced by other factors. The factors that influenced the direction of reading in European languages are not known. However, most people who read also write and until recently, with the advent of typewriters and word processors, writing was first performed on clay tablets with a reed and subsequently with pens and ink. Because $90 \%$ of the population is right handed and write with their right hand, writing from right to left and proximal (bottom) to distal (top) would be more likely to smear the wet clay or ink than writing in the opposite direction. That all current languages, including those Asian languages that are read vertically, are read top down would suggest that there is a strong biological bias. However, evidence against this postulate comes from the observation that Celtic Ogham (Circa $400 \mathrm{CE}$ ) was written bottom up.
This observation provides evidence that factors other than brain-based spatial directional biases influenced the direction of reading and writing. In addition, that Ogham was primarily chiseled into stone provides some evidence that the direction of writing, rather than reading might have influence the top-down direction of reading. The postulate that the direction we use to read might not be predicated on the direction that is most compatible with the attentional biases induced by brain activation, but rather influenced by the writing instruments used by our ancestors write could be tested in children learning to read.

We do not know the reason why we did not find any difference in scanning faces in left versus right hemispace. We expected that, because several studies have suggested that the right hemisphere is dominant for face recognition and has a distal bias, our healthy subjects would have performed more efficiently (faster) when scanning the faces in the left ( $v s$.$) right hemispace and in a top to bottom ( v s$. bottom to top) direction. However, the face search task also required focal attention that, as mentioned, is mediated by the left hemisphere. Hence, the face cancellation task might have activated both hemispheres and therefore did not reveal a hemispace or direction bias.

\section{ACKNOWLEDGMENTS}

This work was partially supported by the Department of Veteran's Affairs and the Lundbeck Pharmaceutical Company. There are not conflicts of interest affecting this manuscript.

\section{REFERENCES}

Arruda, J.E., Amoss, R.T., Coburn, K.L., \& McGee, H. (2007). A quantitative electroencephalographic correlate of sustained attention processing. Applied Psychophysiology and Biofeedback, $32,11-17$.

Arruda, J.E., Weiler, M.D., Valentino, D., Willis, W.G., Rossi, J.S., Stern, R.A., Gold, S.M., \& Costa, L. (1996). A guide or applying principle component analysis and confirmatory factor analysis to quantitative electroencephalogram data. International Journal of Psychophysiology, 23, 63-81.

Barrett, A.M., Beversdorf, D.Q., Crucian, G.P., \& Heilman, K.M. (1998). Neglect after right hemisphere stroke: A smaller floodlight for distributed attention. Neurology, 51, 972-978.

Bowers, D. \& Heilman, K.M. (1980). Pseudoneglect: Effects of hemispace on a tactile line bisection task. Neuropsychologia, 18, 491-498.

Bryden, M.P. \& Allard, F. (1976). Visual hemifield differences depend on typeface. Brain and Language, 3, 191-200.

Cohen, L., Henry, C., Dehaene, S., Martinaud, O., Lehericy, S., Lemer, C., \& Ferrieux, S. (2004). The pathophysiology of letter by letter reading. Neuropsychologia, 42, 1768-1780.

Coslett, H.B. (2003). Acquired dyslexia. In K.M. Heilman \& E. Valenstein (Eds.), Clinical neuropsychology (pp. 108-125). New York: Oxford University Press.

Geldmacher, D.S. \& Heilman, K.M. (1994). Visual field influence on Radial Line bisection. Brain and Cognition, 26, 65-72.

Halligan, P.W. \& Marshall, J.C. (1993). The bisection of horizontal and radial lines: A case study of normal controls and ten 
patients with left visuospatial neglect. International Journal of Neuroscience, 70, 146-167.

Harvey, M., Milner, A.D., \& Roberts, R.C. (1995). An investigation of hemispatial neglect using the landmark task. Brain and Cognition, 27, 59-78.

Heilman, K.M., Chatterjee, A., \& Doty, L.C. (1995). Hemispheric asymmetries of near-far spatial attention. Neuropsychology, 9 , 58-61.

Jager, G. \& Postma, A. (2003). On the hemispheric specialization for categorical and coordinate spatial relations: A review of the current evidence. Neuropsychologia, 41, 504-515.

Jeerakathil, T.J. \& Kirk, A. (1994). A representational vertical bias. Neurology, 44, 703-706.

Jeong, Y., Drago, V., \& Heilman, K.M. (2006). Radial characterline bisection. Cognitive and Behavioral Neurology, 19, $105-108$.

Lamb, M.R. \& Robertson, L.C. (1990). The effect of visual angle on global and local reaction times depends on the set of visual angles presented. Perception and Psychophisic, 47, 489-496.
Lee, G.P., Meador, K.J., Loring, D.W., Allison, J.D., Brown, W.S., Paul, L.K., Pillai, J.J., \& Lavin, T.B. (2004). Neural substrates of emotion as revealed by functional magnetic resonance imaging. Cognitive and Behavioral Neurology, 17, 9-17.

McCourt, M.E., Garlinghouse, M., \& Butler, J. (2001). The influence of viewing eye on pseudoneglect magnitude. Journal of International Neuropsychological Society, 7, 391-395.

Mohr, C. \& Leonards, U. (2007). Rightward bisection errors for letter lines: The role of semantic information. Neuropsychologia, 45, 295-304.

Robertson, L.C., Lamb, M.R., \& Knight, R.T. (1988). Effects of lesions of temporal-parietal junction on perceptual and attentional processing in humans. Journal of Neuroscience, 8 , 3757-3769.

Shelton, P.A., Bowers, D., \& Heilman, K.M. (1990). Peripersonal and vertical neglect. Brain, 113, 191-205.

Weiss, P.H., Marshall, J.C., Wunderlich, G., Tellmann, L., Halligan, P.W., Freund, H.J., Zilles, K., \& Fink, G.R. (2000). Neural consequences of acting in near versus far space: A physiological basis for clinical dissociations. Brain, 12, 2531-2541. 